\title{
The trauma-informed genital and gynecologic examination
}

\author{
Iris Gorfinkel MDCM, Elana Perlow BA, Sheila Macdonald RN MN
}

Cite as: CMAJ 2021 July 19;193:E1090. doi: 10.1503/cmaj.210331

\section{A history of sexual violence reduces the likelihood of people having timely genital or gynecologic examinations \\ In Canada, more than half of transgender people, 1 in 3 women, and 1 in 8 men report experiencing sexual violence. ${ }^{1}$ Genital examinations trigger flashbacks and increase symptoms of anxiety in about $50 \%$ of people who have experienced sexual assault. ${ }^{2,3}$ Given this high prevalence, trauma-informed care starts with the assumption that all people have experienced sexual trauma.}

Trauma-informed examinations are performed "with" patients, ensuring that they feel safe, have control and are offered choices The goal is optimal support while avoiding triggers or exacerbating trauma symptoms. ${ }^{1}$ Health practitioners should use gender-neutral language; be sensitive to cultural, historical and gender issues; and explain to patients what to expect both before and during the examination.

Patient preferences should be established before the

examination and these should be accommodated

Patients may prefer to choose the timing of the examination (e.g., the current health care encounter or a future appointment), and may prefer to have a support person present during the examination. ${ }^{3}$ They may wish to self-collect samples or self-insert instruments.

\section{4.}

\section{Preserving patients' dignity helps them to feel empowered}

during the examination

Practitioners should leave the room when patients disrobe and dress. This validates patient dignity and allows time for them to formulate questions. During the examination, the patient's body should be covered, exposing only those areas it is necessary to expose and for the shortest time required. Findings should be shared only when the patient is fully dressed. ${ }^{4}$

\section{People who decline an examination should be offered self-testing}

Studies of self-testing for human papillomavirus and sexually transmitted infections have shown high rates of participation, ease of self-collection of specimens, and effectiveness of interventions. ${ }^{6}$ Cervical and vaginal selfsampled specimens for Chlamydia trachomatis and Neisseria gonorrhoeae yielded test sensitivities of $94 \%-99 \%$ and specificities of $97 \%-99 \%$; vaginal self-sampled specimens for Candida albicans, dubliniensis, parapsilosis and tropicalis showed test sensitivity of $90 \%-94 \%$ and specificity of $90 \%-93 \%{ }^{6}$ Results of self-collected samples were similar to those from clinician-collected samples. Urine testing is $98 \%$ sensitive and $99 \%$ specific for C. trachomatis and N. gonorrhoeae and may be used instead of cervical swabbing in those who do not wish to collect vaginal specimens. ${ }^{5}$ Kits may be obtained cost free at local laboratories in Canada.

\section{References}

1. Experiences of violent victimization and unwanted sexual behaviours among gay, lesbian, bisexual and other sexual minority people, and the transgender population, in Canada, 2018 [juristat]. Cat no 85-002-X. Ottawa: Statistics Canada; 2020. Available: https://www150.statcan.gc. $\mathrm{ca} / \mathrm{n} 1 / \mathrm{en} /$ pub/85-002-x/2020001/article/00009-eng.pdf?st=W5wDOStd (accessed 2021 June 2).

2. Frecker $\mathrm{H}$, Scheim $\mathrm{A}$, Leonardi $\mathrm{M}$, et al. Experiences of transgender men in accessing care in gynecology clinics [24G]. Obstet Gynecol 2018;131:81S

3. Sexual violence: support and prevention - quick facts. Ottawa: uOttawa. Available: https://www.uottawa.ca/sexual-violence-support -and-prevention/quick-facts (accessed 2021 June 2).

4. Pearl C. trauma-informed pelvic exams. New York: Reproductive Health Access Project; 2015. Available: https://www.reproductiveaccess.org/ resource/trauma-informed-pelvic-exams/ (accessed 2021 June 2).

5. Chlamydia trachomatis and Neisseria gonorrhoeae: nucleic acid amplification testing. Toronto: Public Health Ontario; updated 2019. Available: https://www.publichealthontario.ca/-/media/documents/lab/ lab-sd-005-chlamydia-gonorrhoeae-sensitivity-specificity.pdf?la=en (accessed 2021 June 2).

6. Gaydos CA, Beqaj S, Schwebke JR, et al. Clinical validation of a test for the diagnosis of vaginitis. Obstet Gynecol 2017;130:181-9.

Competing interests: Iris Gorfinkel reports receiving grants from GSK, Merck, Urovant Sciences and Johnson \& Johnson. Between 1991 and 1995, Dr. Gorfinkel received payment for expert testimony for sexual assault service operated through Shaughnessy Hospital and later B.C. Women's Hospital. Dr. Gorfinkel has also received lecture fees from GSK, CME Outfitters and Doctors Nova Scotia; holds stock in Johnson \& Johnson and Merck; and was the co-chair of a Shingrix (GSK) advisory board. Dr. Gorfinkel has contributed to the following in the forms of medical writing and presentations, some with pay: CBC Radio and Television; Zoomer Radio and Television; Global News, CTV News, Bored Panda; The Globe \& Mail; CMAJ; Canadian Family Physician. No other competing interests were declared.

This article has been peer reviewed.

Affiliations: PrimeHealth Clinical Research and Family Practice (Gorfinkel), Toronto, Ont.; Cooper Medical School of Rowan University (Perlow), Camden, NJ; Sexual Assault/ Domestic Violence Care Centre (Macdonald), Toronto, Ont.

Content licence: This is an Open Access article distributed in accordance with the terms of the Creative Commons Attribution (CC BY-NC-ND 4.0) licence, which permits use, distribution and reproduction in any medium, provided that the original publication is properly cited, the use is noncommercial (i.e., research or educational use), and no modifications or adaptations are made. See: https://creativecommons. org/licenses/by-nc-nd/4.0/

Correspondence to: Iris Gorfinkel, i.gor@outlook.com 